\title{
Localization and Delocalization in Dirty Superconducting Wires
}

\author{
P. W. Brouwer, ${ }^{1}$ A. Furusaki, ${ }^{2}$ I. A. Gruzberg, ${ }^{3}$ and C. Mudry ${ }^{4}$ \\ ${ }^{1}$ Laboratory of Atomic and Solid State Physics, Cornell University, Ithaca, New York 14853-2501 \\ ${ }^{2}$ Yukawa Institute for Theoretical Physics, Kyoto University, Kyoto 606-8502, Japan \\ ${ }^{3}$ Institute for Theoretical Physics, University of California, Santa Barbara, California 93106-4030 \\ ${ }^{4}$ Paul Scherrer Institut, CH-5232, Villigen PSI, Switzerland
}

(Received 1 February 2000)

\begin{abstract}
We present Fokker-Planck equations that describe transport of heat and spin in dirty unconventional superconducting quantum wires. Four symmetry classes are distinguished, depending on the presence or absence of time-reversal and spin-rotation invariance. In the absence of spin-rotation symmetry, heat transport is anomalous in that the mean conductance decays like $1 / \sqrt{L}$ instead of exponentially fast for large enough length $L$ of the wire. The Fokker-Planck equations in the presence of time-reversal symmetry are solved exactly and the mean conductance for quasiparticle transport is calculated for the crossover from the diffusive to the localized regime.
\end{abstract}

PACS numbers: 72.15.Rn, 73.20.Fz, 73.23.-b, 74.25.Fy

The discovery of the $d$-wave nature of the order parameter in high $T_{c}$ materials has renewed interest in unconventional superconductors with low energy quasiparticles near the Fermi energy $\varepsilon_{F}$. An important question is how disorder affects the quasiparticle dynamics and the corresponding low-temperature properties of the superconductor. In Ref. [1] it is predicted that, on energy scales $\left|\varepsilon-\varepsilon_{F}\right|$ less than the inverse mean free time and on length scales beyond the mean free path, weak impurity scattering leads to a finite density of states (DOS) and to a diffusive dynamics of quasiparticles. In normal metals, it has been known for a long time that quantum interference imposes corrections to this picture, in the form of weak localization, and eventually, for dimensions $\leq 2$, exponential (Anderson) localization. The analogous question for low-energy quasiparticles in unconventional superconductors has been considered only recently [2-7].

The crucial distinction between quasiparticles in a superconductor and in a normal metal, is that the former are described by a Hamiltonian of Bogoliubov-de Gennes (BdG) type. Such a Hamiltonian has an additional particle-hole grading, accompanied by a discrete particlehole symmetry, which is absent in the Hamiltonian for (electronlike) quasiparticles in a normal metal. Symmetry plays a crucial role in the problem of Anderson localization. A classification of the symmetry classes for BdG Hamiltonians, depending on the presence or absence of time-reversal (TR) and spin-rotation (SR) symmetry, has been given by Altland and Zirnbauer [8]. The four possibilities are denoted C, CI, D, and DIII; see Table I. Reference [8] addressed the "zero-dimensional" (0D) case of chaotic quantum dots with superconducting leads. The higher dimensional realizations of the BdG symmetry classes, relevant for the question of localization, were studied in Refs. [2-7], mainly by field-theoretical methods involving construction and analysis of nonlinear sigma models with appropriate symmetries.
In this Letter, we study localization in the BdG symmetry classes for the geometry of a quantum wire, i.e., in quasi-one-dimension (quasi-1D). For this purpose, we use the Fokker-Planck (FP) approach $[9,10]$, which is complementary to the nonlinear sigma model of Refs. [2-4,6,7]. Using the classification scheme of Ref. [8], we obtain four FP equations that control quasiparticle transport at the Fermi level in a dirty superconducting wire. Our findings are remarkable: While for classes $\mathrm{C}$ and $\mathrm{CI}$ the mean and typical values of the quasiparticle conductance $g$ decay exponentially with the length $L$ of the wire for large $L$, the situation in classes D and DIII is quite different. There the mean $\langle g\rangle$ decays only algebraically to zero for large $L$ and $\ln g$ is not self-averaging, indicating a very broad distribution of the conductance and the absence of the exponential localization of the quasiparticle states at $\varepsilon_{F}$. (The absence of exponential localization for class $\mathrm{D}$ has been announced independently in Ref. [7].)

It should be stressed that the BdG Hamiltonians do not conserve charge. Instead, the conserved densities are those of the energy (in all four classes) and spin (when the SR symmetry is present). Thus, the transport properties (the conductance $g$ ) studied in this Letter refer to transport of heat and spin.

We now proceed with a detailed statement of our results and their derivation. The model that we consider is that of a disordered quantum wire, with a Hamiltonian of the $\mathrm{BdG}$ form. We distinguish gradings corresponding to spin up/down, particle/hole, left/right movers. Denoting these with Pauli matrices $\sigma, \gamma$, and $\tau$, respectively, we write our model Hamiltonian as

$$
\mathcal{H}=\mathcal{K}+\mathcal{V}, \quad \mathcal{K}=i v_{F} \partial_{x} \sigma_{0} \otimes \gamma_{0} \otimes \tau_{3} \otimes \mathbb{1}_{N}
$$

where $\sigma_{0}$ is the $2 \times 2$ unit matrix in the spin grading, etc. The kinetic energy $\mathcal{K}$ describes the propagation of right and left moving quasiparticles in $N$ channels at the Fermi 
TABLE I. Definition of the symmetry classes for a dirty superconducting quantum wire, with respect to the presence of spin-rotation (SR) and time-reversal (TR) symmetry. Listed here are the Lie group $\mathcal{L}$ of the transfer matrix $\mathcal{M}$, the factor group $\mathcal{G}$ of angular degrees of freedom of $\mathcal{M}$, the multiplicities of the roots of the symmetric space $\mathcal{L} / \mathcal{G}$, and the degeneracies of the radial coordinates $x_{j}$ of the transfer matrix $\mathcal{M}$.

\begin{tabular}{cccccccr}
\hline \hline Class & SR & TR & $\mathcal{L}$ & $G$ & $m_{0}$ & $m_{l}$ & $d$ \\
\hline $\mathrm{C}$ & Yes & No & $\mathrm{Sp}(N, N)$ & $\mathrm{Sp}(N) \times \mathrm{Sp}(N)$ & 4 & 3 & 4 \\
$\mathrm{CI}$ & Yes & Yes & $\mathrm{Sp}(N, \mathbb{C})$ & $\mathrm{Sp}(N)$ & 2 & 2 & 4 \\
$\mathrm{D}$ & No & No & $\mathrm{O}(4 N, 4 N)$ & $\mathrm{O}(4 N) \times \mathrm{O}(4 N)$ & 1 & 0 & 1 \\
DIII & No & Yes & $\mathrm{O}(4 N, \mathbb{C})$ & $\mathrm{O}(4 N)$ & 2 & 0 & 2 \\
\hline \hline
\end{tabular}

level. The "potential" $\mathcal{V}(x)$ is an $8 N \times 8 N$ matrix that accounts both for the presence of disorder and of superconducting correlations. In particle/hole $(\gamma)$ grading it reads

$$
\mathcal{V}=\left(\begin{array}{cc}
v & \Delta \\
-\Delta^{*} & -v^{\mathrm{T}}
\end{array}\right)
$$

where $v(\Delta)$ is a Hermitian (antisymmetric) $4 N \times 4 N$ matrix, representing the impurity potential (superconducting order parameter). The form (2) of the potential $\mathcal{V}$ ensures that the Hamiltonian $\mathcal{H}$ obeys particle-hole symmetry, $\mathcal{H}=-\gamma_{1} \mathcal{H}^{\mathrm{T}} \gamma_{1}$ [8]. In addition, $\mathcal{H}$ (and hence $\mathcal{V}$ ) may obey TR invariance $\mathcal{H}=\mathcal{T} \mathcal{H}^{*} \mathcal{T}^{-1}$, with $\mathcal{T}=i \tau_{1} \otimes \sigma_{2}$, and/or SR invariance $\mathcal{H}=-\gamma_{2} \mathcal{H}^{\mathrm{T}} \gamma_{2}$.

Spatial fluctuations of the order parameter $\Delta$ and the potential $v$ are taken into account by assuming that $\mathcal{V}$ is a Gaussian random variable with vanishing mean, i.e. that its probability functional $P[V]$ is of the form

$$
P[\mathcal{V}] \propto \exp \left[-\frac{\gamma \ell}{4 c} \int_{0}^{L} d x \operatorname{tr} \mathcal{V}^{2}(x)\right],
$$

where $\ell$ is the mean free path, $\gamma$ is a numerical constant to be defined below, and $c=1$ (2) for class C/D (CI/DIII). The transport properties of the Hamiltonian $\mathcal{H}$ describe the transport of spin and heat by quasiparticles in a disordered superconducting quantum wire.

Before we continue with the analysis of our model (1)-(3), some remarks about its validity and relevance are in place. One key property of the model is that, apart from corrections at very low energies due to quasiparticle localization [4] or the appearance of a critical state, the DOS of the Hamiltonian $\mathcal{H}$ near the Fermi level $\varepsilon_{F}$ is nonzero and finite. This is related to the fact that the statistical average of the order parameter $\Delta$ is zero in our model, cf. Eq. (3). For a dirty superconductor, such behavior is plausible if the order parameter is unconventional, as in $d$-wave superconductors, or when it breaks TR symmetry, as is believed to be the case for, e.g., the ruthenates [11], vortex lines in a (conventional) superconductor [3], or a normal metal wire with magnetic impurities that is weakly connected to a superconducting substrate. In all these cases the disorder leads to the existence of low-energy quasiparticle states $[1,4]$. The Hamiltonian (1) then describes diffusion and localization of these "disorder-facilitated" quasiparticles. An altogether different scenario is that of a wire made out of an unconventional superconductor with very weak disorder. If boundary conditions are suitably chosen, one or several propagating modes can exist at $\varepsilon_{F}$, whose localization properties are described by Eq. (1). In any case, one should view $\mathcal{H}$ as an effective or coarse grained Hamiltonian, whose validity is restricted to length scales beyond the microscopic mean free path $\ell$. It is universal in the sense that its form is determined solely by the symmetry, and the distribution (3) provides for the existence of the diffusive regime with a finite DOS at the proper energy scale. (Note that the restrictions to the validity of our model are not different from those of related field theoretic descriptions appearing in the literature $[3,4,6,7]$.)

We describe transport properties of the model (1)-(3) through its $8 N \times 8 N$ transfer matrix $\mathcal{M}$ that encodes the $x$ dependence of an $8 N$-component quasiparticle wave function $\psi$ satisfying the Schrödinger equation $\mathcal{H} \psi=$ $\varepsilon \psi$ at $\varepsilon=0, \psi(x+L)=\mathcal{M}(x+L, x) \psi(x)$. Formally, $\mathcal{M}$ is related to the Hamiltonian (1) as

$$
\mathcal{M}(x+L ; x)=T_{y} \exp \left[i \int_{x}^{x+L} d y \tau_{3} \mathcal{V}(y)\right],
$$

where $T_{y}$ denotes the path ordering operator for the $y$ integration along the wire. From Eq. (4) one finds that flux conservation (i.e., Hermiticity of $\mathcal{H}$ ) and particle-hole symmetry imply that $\mathcal{M}^{\dagger} \tau_{3} \mathcal{M}=\tau_{3}$ and $\gamma_{1} \mathcal{M} \gamma_{1}=\mathcal{M}^{*}$, respectively. Further, TR invariance requires $\mathcal{T} \mathcal{M} \mathcal{T}^{-1}=\mathcal{M}^{*}$, while $\mathrm{SR}$ invariance is obeyed if $\gamma_{2} \mathcal{M} \gamma_{2}=\mathcal{M}^{*}$. The transfer matrix $\mathcal{M}$ obeys the multiplicative rule $\mathcal{M}(z, x)=\mathcal{M}(z, y) \mathcal{M}(y, x)$ for $x<y<z$ and hence is an element of a certain Lie group $\mathcal{L}$. The appropriate Lie groups for the four symmetry classes are listed in Table I. We note that the actual transfer matrix group is an $8 \mathrm{~N}$-dimensional representation of the Lie group $\mathcal{L}$, where $\mathcal{L}$ also allows a lower dimensional (irreducible) representation for the classes $\mathrm{C}, \mathrm{CI}$, and DIII [12]. Elements of $\mathcal{L}$ are conveniently parametrized in terms of their polar decomposition, which, in an irreducible representation, takes the form

$$
\begin{gathered}
\left(\begin{array}{cc}
V_{1} & 0 \\
0 & V_{2}
\end{array}\right)\left(\begin{array}{cc}
\cosh X & \sinh X \\
\sinh X & \cosh X
\end{array}\right)\left(\begin{array}{cc}
V_{3} & 0 \\
0 & V_{4}
\end{array}\right) \quad(\mathrm{C}, \mathrm{D}), \\
V_{1}\left(\begin{array}{cc}
\cosh X & i \sinh X \\
-i \sinh X & \cosh X
\end{array}\right) V_{2} \quad(\mathrm{CI}, \mathrm{DIII}) .
\end{gathered}
$$

Here $V_{i} \in \mathrm{O}(4 N)[\mathrm{Sp}(N)]$ for classes D/DIII [C/CI], for all $i=1,2,3,4$, and $X$ is a diagonal matrix with positive entries $x_{j}$. (By Kramers' degeneracy, the elements of $X$ 
occur in pairs in class C.) The $x_{j}$ serve as radial coordinates on the Lie Group $\mathcal{L}$. One verifies that the eigenvalues of the true $8 N \times 8 N$ transfer matrix $\mathcal{M M}^{\dagger}$ occur in $d$-fold degenerate inverse pairs $\exp \left( \pm 2 x_{j}\right)$, where the degeneracy $d$ is listed in Table I. Hence the number of independent $x_{j}$ 's is $4 N / d$. Finally, we note that the $x_{j}$ are related to the conductance $g$ through [10]

$$
g=d \sum_{j=1}^{4 N / d} \cosh ^{-2} x_{j} .
$$

Our aim is to find the probability distribution of the $x_{j}$ for a transfer matrix corresponding to the model (1)-(3). Increasing the length $L$ of the wire by a small increment $\delta L$ amounts to multiplication of its transfer matrix $\mathcal{M}(L)=\mathcal{M}(x+L, x)$ by a transfer matrix $\mathcal{M}^{\prime}=$ $\mathcal{M}(x+L+\delta L, x+L)$. Since $\mathcal{M}^{\prime}$ is close to the unit matrix, random, and statistically independent from $\mathcal{M}(L)$, we find that as a function of $L, \mathcal{M}(L)$ performs a random trajectory on its Lie group $\mathcal{L}$. Actually, we do not need to know the full trajectory on $\mathcal{L}$ if we are only interested in the conductance $g$. It is sufficient to know the trajectory of the radial coordinates $x_{j}$ of $\mathcal{M}(L)$ after dividing out a maximal compact subgroup $G$ of $\mathcal{L}$ corresponding to the angular degrees freedom of $\mathcal{M}$ that leave the product $\mathcal{M} \mathcal{M}^{\dagger}$ invariant, or, in other words, to know the trajectory of the $x_{j}$ in the symmetric space $\mathcal{L} / \mathcal{G}$ [13]. The subgroups $G$ are listed in Table I. Starting from the microscopic model (1)-(3), one can show that the trajectory obeyed by the $x_{j}$ is a Brownian motion on the coset space $\mathcal{L} / \mathcal{G}$ described by the joint probability distribution $P\left(x_{1}, \ldots, x_{4 N / d} ; L\right)$. The $L$ evolution of $P$ is described by a FP equation, which follows either from a direct calculation starting from Eq. (1), or from the general theory of symmetric spaces [13]. In both cases we find

$$
\begin{aligned}
\frac{\partial P}{\partial L} & =\frac{1}{2 \gamma \ell} \sum_{j=1}^{4 N / d} \frac{\partial}{\partial x_{j}}\left[J\left(\frac{\partial}{\partial x_{j}} J^{-1} P\right)\right], \\
J & =\prod_{j=1}^{4 N / d}\left|\sinh 2 x_{j}\right|^{m_{l}} \prod_{k>j}^{4 N / d} \prod_{ \pm}\left|\sinh \left(x_{j} \pm x_{k}\right)\right|^{m_{o}},
\end{aligned}
$$

where the numbers $m_{l}$ and $m_{o}$ are the long and ordinary root multiplicities in the symmetric spaces $\mathcal{L} / G$, see Table I, and $\gamma=\left(4 N m_{o} / d\right)+1-m_{o}+m_{l}$. The FP equation (6) is supplemented with the boundary condition $\partial P / \partial x_{j}=(P / J) \partial J / \partial x_{j}$ at $x_{j}=0$. The initial condition $\mathcal{M}=1$ for $L=0$ corresponds to $P\left(x_{1}, \ldots, x_{4 N / d} ; 0\right)=$ $\prod_{j} \delta\left(x_{j}\right)$.

The FP equation (6) is the fundamental equation that governs quasiparticle transport and localization in quantum wires of the symmetry classes C, CI, D, and DIII.

In the localized regime $L \gg N \ell$, typically all $x_{j}$ and their spacings are much bigger than unity, and the conductance is governed by the smallest coordinate $x_{1}$. That coordinate has a Gaussian distribution, with mean $m_{l} L / \gamma \ell$ and variance $L / \gamma \ell$. For classes $\mathrm{C}$ and $\mathrm{CI}$ this implies that $g$ is exponentially small, with

$$
\langle\ln g\rangle=-\frac{2 m_{l} L}{\gamma \ell}, \quad \operatorname{var} \ln g=\frac{4 L}{\gamma \ell},
$$

with $m_{l}=2$ for class CI and $m_{l}=3$ for class C. Exponential localization for class $\mathrm{C}$ in quasi-1D was previously obtained by Bundschuh et al. [3], using the nonlinear sigma model. For class D and DIII, however, $m_{l}=0$, so that there is no exponential localization. Instead, $g$ has a very broad distribution (broader than log-normal), with an algebraic decay of the mean and the variance and an $L^{1 / 2}$ dependence of $\ln g$,

$$
\begin{gathered}
\langle g\rangle=d \sqrt{\frac{2 \gamma \ell}{\pi L}}, \quad \operatorname{var} g=\frac{2 d}{3}\langle g\rangle, \\
\langle\ln g\rangle=-4 \sqrt{\frac{L}{2 \pi \gamma \ell}}, \quad \operatorname{var} \ln g=\frac{4(\pi-2) L}{\pi \gamma \ell} .
\end{gathered}
$$

Hence in classes D and DIII, quasiparticle states are not localized at the Fermi level. Since they are neither truly extended (typically $g \ll 1$ in class D and DIII), we label them critical, following terminology from the case of quantum wires with off-diagonal disorder, where similar behavior is found at the center of the band $[14,15]$.

The effect of disorder is much less pronounced in the diffusive regime $\ell \ll L \ll N \ell$. Here, the conductance has only small fluctuations around its mean. Following the method of moments [10], we find $\langle g\rangle$ from the FP equation by construction of evolution equations for the moments of $g_{a}=d \sum_{j} \cosh ^{-2 a} x_{j}, a=1,2, \ldots$,

$$
\begin{aligned}
\frac{\gamma \ell}{a} \frac{\partial\left\langle g_{a}\right\rangle}{\partial L}= & \frac{m_{o}}{d} \sum_{n=1}^{a-1}\left\langle g_{a-n} g_{n}\right\rangle-\frac{m_{o}}{d} \sum_{n=1}^{a}\left\langle g_{a-n+1} g_{n}\right\rangle \\
& +\left(a m_{o}-2 a-1+m_{l}\right)\left\langle g_{a+1}\right\rangle \\
& +\left(2 a-a m_{o}+m_{o}-2 m_{l}\right)\left\langle g_{a}\right\rangle
\end{aligned}
$$

In the diffusive regime one may replace the average of a product by the product of the averages, and hence one finds for $\ell \ll L \ll N \ell$

$$
\langle g\rangle=\frac{4 N \ell}{L+\ell}+\frac{d\left(m_{o}-2 m_{l}\right)}{3 m_{o}}+\mathcal{O}(\ell / L, L / N \ell) .
$$

The first term in Eq. (10) is the Drude conductance, while the second term is the first quantum interference correction to the average conductance. For classes C, CI, D, and DIII it takes the values $-2 / 3,-4 / 3,1 / 3$, and $2 / 3$, respectively. The weak localization correction for class $\mathrm{C}$ was obtained earlier in Ref. [3]. For the classes D and DIII, the correction is positive, i.e., quantum interference enhances the conductance relative to the classical Drudelike leading behavior (see also Ref. [3]). This is similar to the phenomenon of antilocalization in the standard symplectic symmetry class, though, as pointed out by Bocquet et al. [7], here it is a precursor of the breakdown of exponential localization, while in the standard symplectic class localization takes over in higher order quantum corrections. 
In the presence of TR symmetry, Eq. (6) is soluble. This is in contrast to the case of the FP equations for the standard and chiral symmetry classes, where only the case of broken TR symmetry was exactly solvable [10,15]. The first step [10] is a map of Eq. (6) onto a Schrödinger equation in imaginary time for the wave function $\Psi\left(\left\{x_{j}\right\} ; s\right)=\exp \left[-\frac{1}{2} \ln J\left(\left\{x_{j}\right\}\right)\right] P\left(\left\{x_{j}\right\} ; s\right)$ for $4 N / d$ fermions in one dimension with coordinates on the halfline $x>0$. They interact through a two-body potential proportional to $m_{o}-2$ in the presence of a one-body potential proportional to $\left(m_{l}-2\right) m_{l}$. Hence, for classes DIII and CI these fermions are free and they differ only by the boundary condition obeyed by their wave functions $\Psi$ at the origin. We thus find the solutions

$$
\begin{aligned}
P \propto & \prod_{j}\left(x_{j} \sinh 2 x_{j}\right)^{m_{l} / 2} e^{-\gamma x_{j}^{2} \ell / 2 L} \\
& \times \prod_{j<k}\left(x_{k}^{2}-x_{j}^{2}\right)\left(\sinh ^{2} x_{k}-\sinh ^{2} x_{j}\right) .
\end{aligned}
$$

Using the method of biorthogonal functions [16] it is then possible to calculate the average conductance $\langle g\rangle$ for all $N$ and $L$. Here we report the result for the limit of large $N$, leaving the results for finite $N$ for a future publication,

$$
\begin{aligned}
& \langle g\rangle=\frac{1}{s}-\frac{4}{3}+4 \sum_{n=1}^{\infty} e^{-\pi^{2} n^{2} / 4 s}\left(\frac{1}{s}+\frac{2}{\pi^{2} n^{2}}\right) \\
& \langle g\rangle=\frac{1}{s}+\frac{2}{3}-4 \sum_{n=1}^{\infty} e^{-\pi^{2} n^{2} / 2 s} \frac{1}{\pi^{2} n^{2}} \quad \text { DIII, }
\end{aligned}
$$

where $s=L /(4 N \ell)$. Note the agreement with Eq. (10) in the diffusive regime $s \ll 1$. In the localized regime $s \gg 1$, Eq. (12) may be resummed, and the asymptotic result (8) is reproduced for class DIII, while for class CI one finds $\langle g\rangle=8(\pi s)^{-1 / 2} \exp (-4 s)$. Quite remarkably, the exact results (12) for $\langle g\rangle$ in classes CI and DIII are related to the average conductance $\langle g\rangle_{\mathrm{ch}}$ in the chiral unitary ensemble for odd channel number [15] as $\langle g(s / 2)\rangle_{\mathrm{CI}}+$ $2\langle g(s)\rangle_{\text {DIII }}=4\langle g(s)\rangle_{\mathrm{ch}}$.

The absence of localization in wires of classes D and DIII may have important implications for higher dimensions, provided our results can be extended beyond $1 \mathrm{D}$, and provided they are not restricted to the regime of weak disorder. With respect to the latter restriction, we can point to the close formal similarity of the delocalization for the FP equations of class D/DIII and the corresponding FP equation for the chiral symmetry classes with odd $N$, where it is understood that the absence of localization holds both for weak and strong disorder [14,17]. Thus arguing that quasiparticle states at the Fermi level remain delocalized for arbitrary disorder strength and dimensionality in the $\mathrm{D}$ classes, our result suggests a possible resolution of a controversy in the literature surrounding 2D disordered superconductors of class D [6,7]. While all Refs. [6,7] assumed existence of two localized phases, distinguished by the quantized value of the Hall conductivity $\sigma_{x y}$, and a metallic phase, the proposed global phase diagrams and transitions between the phases differ considerably. We suggest that the solution might simply lie in the absence of localized phases for classes D and DIII in any dimension $\geq 1$.

In conclusion, we considered quasiparticle transport and localization in disordered quasi-1D superconducting wires at the Fermi level for the four Bogoliubov-de Gennes symmetry classes C, CI, D, and DIII. We obtained and solved the Fokker-Planck equations for the probability of the radial coordinates of the transfer matrix. While quasiparticle states are localized in classes C/CI, localization is absent if spin-rotation symmetry is broken (classes D/DIII).

We thank A. Altland, L. Balents, M.P. A. Fisher, N. Read, T. Senthil, and M. Sigrist for valuable discussions. P.W.B. gratefully acknowledges that this problem was suggested to him in an earlier stage by A. Altland. Close to completion of this work, we learned that J. T. Chalker and co-workers obtained independently similar results for class D; see also Ref. [7]. This work was supported by a Grant-in-Aid for Scientific Research from the Japan Society for the Promotion of Science No. 11740199 (A. F.), and by the NSF under Grant No. DMR-9528578 (I. A. G.).

[1] L. P. Gorkov and P. A. Kalugin, JETP Lett. 41, 253 (1985); S. Schmitt-Rink et al., Phys. Rev. Lett. 57, 2575 (1986); P. A. Lee, ibid. 71, 1887 (1993).

[2] A. Altland et al., JETP Lett. 67, 22 (1998).

[3] R. Bundschuh et al., Nucl. Phys. B532, 689 (1998); Phys. Rev. B 59, 4382 (1999).

[4] T. Senthil et al., Phys. Rev. Lett. 81, 4704 (1998); T. Senthil and M. P. A. Fisher, Phys. Rev. B 60, 6893 (1999).

[5] V. Kagalovsky et al., Phys. Rev. Lett. 82, 3516 (1999); T. Senthil et al., Phys. Rev. B 60, 4245 (1999); I. A. Gruzberg et al., Phys. Rev. Lett. 82, 4524 (1999); Y. Morita and Y. Hatsugai, cond-mat/9907001.

[6] T. Senthil and M.P. A. Fisher, Phys. Rev. B 61, 9690 (2000); N. Read and D. Green, Phys. Rev. B 61, 10267 (2000).

[7] M. Bocquet et al., cond-mat/9910480.

[8] A. Altland and M. R. Zirnbauer, Phys. Rev. Lett. 76, 3420 (1996); Phys. Rev. B 55, 1142 (1997).

[9] O. N. Dorokhov, JETP Lett. 36, 318 (1982); P. A. Mello et al., Ann. Phys. (N.Y.) 181, 290 (1988).

[10] C. W. J. Beenakker, Rev. Mod. Phys. 69, 731 (1997).

[11] G. M. Luke et al., Nature (London) 394, 558 (1998); M. Sigrist et al., Physica (Amsterdam) 317C-318C, 134 (1999).

[12] For instance, in the presence of $\mathrm{SR}$ invariance, $\mathcal{M}$ separates into two identical blocks of size $4 N \times 4 N$.

[13] A. Hüffmann, J. Phys. A 23, 5733 (1990); M. Caselle, cond-mat/9610017.

[14] F. J. Dyson, Phys. Rev. 92, 1331 (1953); A. D. Stone and J. D. Joannopoulos, Phys. Rev. B 24, 3592 (1981).

[15] P. W. Brouwer et al., Phys. Rev. Lett. 81, 862 (1998); C. Mudry et al., Phys. Rev. B 59, 13221 (1999).

[16] K. A. Muttalib, J. Phys. A 28, L159 (1995); K. Frahm, Phys. Rev. Lett. 74, 4706 (1995).

[17] D. S. Fisher, Phys. Rev. B 50, 3799 (1994); 51, 6411 (1995). 